\title{
A relação dos sintomas de bruxismo e disfunção temporomandibular e a ansiedade ocasionada pela pandemia da COVID-19: uma revisão de literatura
}

\author{
The relationship between bruxism symptoms and temporomandibular disorders and anxiety \\ caused by the COVID-19 pandemic: a literature review \\ La relación entre los síntomas del bruxismo y los trastornos temporomandibulares y la ansiedad \\ causada por la pandemia COVID-19: una revisión de la literatura
}

\author{
Erika Thaís Cruz da Silva \\ ORCID: https://orcid.org/0000-0002-8475-7929 \\ Universidade Estadual da Paraíba, Brasil \\ E-mail: erika-thais-silva@ hotmail.com \\ Adriano Flores da Silva \\ ORCID: https://orcid.org/0000-0001-7515-4088 \\ Universidade Estadual da Paraíba, Brasil \\ E-mail: floresuepb@gmail.com \\ Aretha Helen Aragão Lourenço \\ ORCID: https://orcid.org/0000-0001-8851-3473 \\ Universidade Estadual da Paraíba, Brasil \\ E-mail: arethaaragao6@gmail.com \\ Ari Dantas de Carvalho Júnior \\ ORCID: https://orcid.org/0000-0002-9068-7058 \\ Universidade Estadual da Paraíba, Brasil \\ E-mail: dantasd46@gmail.com \\ Nilson Emmanuel Gomes Pereira \\ ORCID: https://orcid.org/0000-0002-4974-7119 \\ Universidade Estadual da Paraíba, Brasil \\ E-mail: nilsonegp@gmail.com \\ Priscila Lima Bezerra \\ ORCID: https://orcid.org/0000-0003-2458-498X \\ Universidade Estadual da Paraíba, Brasil \\ E-mail: eupriscilalimabe@gmail.com \\ Suellen Rabelo Rocha da Costa \\ ORCID: https://orcid.org/0000-0003-0841-2061 \\ Universidade Estadual da Paraíba, Brasil \\ E-mail: suellenrabelo@hotmail.com.br
}

\begin{abstract}
Resumo
O objetivo do presente trabalho foi realizar uma revisão de literatura acerca A relação dos sintomas de bruxismo e disfunção temporomandibular e a ansiedade ocasionada pela pandemia de COVID-19. Foi realizada uma revisão de estudos disponíveis na literatura por meio da busca bibliográfica nas bases de dados eletrônicos Pubmed/Medline e Google acadêmico. Para a pesquisa foram utilizados os seguintes descritores: "Pandemic" AND "Covid-19"AND "Bruxism" AND "Temporomandibular disorder". Outra estratégia de pesquisa foi à busca manual em listas de referências dos artigos selecionados. Como critérios de inclusão, foram adotados os artigos escritos em inglês e português; aqueles que se enquadravam no enfoque do trabalho e os mais relevantes em termos de delineamento das informações desejadas. As consequências globais da pandemia de COVID-19 são diversas e envolvem também o aspecto psicológico. A pandemia da COVID-19 pode estar associada ao aumento de sintomas psicológicos como ansiedade e depressão em virtude do período de isolamento social. Esses sintomas possuem associação as disfunções temporomandibulares e bruxismo levando ao surgimento e desenvolvimento de ambas afecções.
\end{abstract}

Palavras-chave: COVID-19; Pandemia; Bruxismo; Disfunção da articulação temporomandibular.

\begin{abstract}
The aim of the present study was to perform a literature review about the relationship between the symptoms of bruxism and temporomandibular disorders and the anxiety caused by the pandemic of COVID-19. A review of studies available in the literature was performed through bibliographic search in the electronic databases Pubmed / Medline and Google academic. The following descriptors were used for the research: "Pandemic" AND "Covid-19" AND "Bruxism" AND "Temporomandibular disorder". Another search strategy was to manually search the reference lists
\end{abstract}


of selected articles. As inclusion criteria, articles written in English and Portuguese were adopted; those that fit the work focus and the most relevant in terms of outlining the desired information. The global consequences of the COVID-19 pandemic are diverse and also involve the psychological aspect. The COVID-19 pandemic may be associated with an increase in psychological symptoms such as anxiety and depression due to the period of social isolation. These symptoms are associated with temporomandibular disorders and bruxism leading to the appearance and development of both conditions.

Keywords: COVID-19; Pandemics; Bruxism; Temporomandibular joint dysfunction.

\section{Resumen}

El objetivo del presente estudio fue realizar una revisión de la literatura sobre la relación entre los síntomas del bruxismo y los trastornos temporomandibulares y la ansiedad provocada por la pandemia de COVID-19. Se realizó una revisión de los estudios disponibles en la literatura mediante búsqueda bibliográfica en las bases de datos electrónicas Pubmed / Medline y Google Academic. Para la investigación se utilizaron los siguientes descriptores: "Pandemia" Y "Covid-19" Y "Bruxismo" Y "Trastorno temporomandibular". Otra estrategia de búsqueda fue buscar manualmente las listas de referencias de los artículos seleccionados. Como criterio de inclusión, se adoptaron artículos escritos en inglés y portugués; los que se ajustan al enfoque del trabajo y los más relevantes en cuanto a delinear la información deseada. Las consecuencias globales de la pandemia de COVID-19 son diversas y también involucran el aspecto psicológico. La pandemia de COVID-19 puede estar asociada con un aumento de síntomas psicológicos como ansiedad y depresión debido al período de aislamiento social. Estos síntomas están asociados con trastornos temporomandibulares y bruxismo que conducen a la aparición y desarrollo de ambas afecciones.

Palabras clave: COVID-19; Pandemia; Bruxismo; Disfunción de la articulación temporomandibular.

\section{Introdução}

A pandemia de COVID-19 iniciou na cidade de Wuhan, na China, em dezembro de 2019, após diversos pacientes serem diagnosticados com uma pneumonia ocasionada pelo novo coronavírus (Li et al., 2020; Emodi-Perlman et al. 2020). A Organização Mundial de Saúde (OMS) anunciou o surto de COVID-19 como uma pandemia em março de 2020 constituindo uma emergência de saúde pública de interesse internacional (OMS, 2020).

As consequências globais da pandemia de COVID-19 são diversas e envolvem também o aspecto psicológico. Esse alto impacto nas questões psicológicas foi documentado em um estudo desenvolvido na China, onde uma parcela significativa da população relatou ansiedade moderada a grave (Almeida-Leite et al., 2020).

Em uma reportagem veiculado pelo jornal "O Estadão" em outubro de 2020 foi informado sobre o aumento nos casos de bruxismo e fraturas dentárias relatados pelos dentistas associando-os à ansiedade provocada pela quarentena. A maioria desses casos foi atendida pela rede privada de consultórios tendo em vista que no sistema público o atendimento ficou bastante restrito tendo uma queda no número de atendimentos em $80 \%$ comparado ao período anterior à pandemia (O Estadão, 2020).

É bem estabelecida na literatura a associação entre fatores psicossociais e o desenvolvimento das Disfunções Temporomandibulares (DTM). Alguns autores também associam o Bruxismo a questões psicológicas (Almeida-Leite et al., 2020). Em um estudo realizado por Quadri et al. (2015) foi relatado que pacientes com altos níveis de estresse possuem seis vezes mais chances de relatarem sintomas de bruxismo.

Diante do exposto acima, o presente trabalho tem como objetivo realizar uma revisão de literatura acerca da relação dos sintomas de bruxismo e disfunção temporomandibular e a ansiedade ocasionada pela pandemia de COVID-19.

\section{Metodologia}

Foi realizada uma revisão de estudos disponíveis na literatura por meio da busca bibliográfica nas bases de dados eletrônicos Pubmed/Medline e Google acadêmico. Para a pesquisa foram utilizados os seguintes descritores: "Pandemic" AND “Covid-19”AND "Bruxism” AND “Temporomandibular disorder”. Outra estratégia de pesquisa foi à busca manual em listas de referências dos artigos selecionados. Como critérios de inclusão, foram adotados os artigos escritos em inglês e português; 
aqueles que se enquadravam no enfoque do trabalho e os mais relevantes em termos de delineamento das informações desejadas.

Foram observados e determinados alguns aspectos para a inclusão dos estudos na revisão; como a significância, a confiabilidade e clareza no detalhamento metodológico das informações apresentadas. Assim como, foi indispensável à disponibilidade do texto integral para sua inclusão no estudo. Foram excluídos da amostra os artigos que não apresentaram relevância sobre o tema abordado.

\section{Revisão de Literatura}

O bruxismo consiste num hábito parafuncional onde o paciente possui o hábito de ranger os dentes e é definido por alguns estudiosos como "atividade muscular repetitiva da mandíbula caracterizada por apertar ou ranger os dentes e/ ou por imobilização ou projeção da mandíbula". O bruxismo pode ser classificado em bruxismo do sono e bruxismo acordado (Machado et al., 2020; Quadri et al., 2015; Przystańska et al., 2019).

O bruxismo acordado é representa uma atividade muscular mastigatória que ocorre durante a vigília e possui como características principais $r$ contato repetitivo ou sustentado dos dentes e/ou por imobilização ou impulso da mandíbula, além disso, não é considerado distúrbio do movimento em indivíduos saudáveis (Machado et al., 2020).

A prevalência de "bruxismo do sono" varia de $9,3 \%$ a 15,9\% e a prevalência de "bruxismo acordado" varia de $22,1 \%$ a $31 \%$ na população adulta. Acredita-se que a presença de comorbidades, como outras doenças psicológicas, podem influenciar na avaliação da prevalência de bruxismo (Przystańska et al., 2019).

As disfunções temporomandibulares (DTM) representam um grupo de afecções que causam dor e disfunção dos músculos mastigatórios, das articulações temporomandibulares (ATMs) e outras estruturas. As características mais comuns da DTM são dor regional, limitação dos movimentos mandibulares e ruídos das ATMs durante os movimentos. São mais frequentes em mulheres do que em homens e possuem como fatores etiológicos alguns fatores psicossociais, como ansiedade, estresse e depressão (Emodi-Perlman et al., 2020).

As DTMs podem conduzir também ao desenvolvimento de sinais e sintomas associados, que não estão relacionados diretamente ao sistema musculoesquelético, como zumbido, otalgia referida, cefaléia (cefaléia tensional ou enxaqueca), cervical e miofascial (Kmeid et al., 2020).

Os fatores etiológicos da disfunção da articulação temporomandibular são os biológicos, ambientais (tabagismo), emocionais (depressão e ansiedade), sociais e cognitivos. Além disso, existe uma associação com outras condições que causam dor, como dores de cabeça crônicas, fibromialgia, distúrbios autoimunes (como síndrome de Sjögren, artrite reumatóide (Kmeid et al., 2020).

De acordo com relatório da Organização Mundial da Saúde (OMS), a DTM é a terceira desordem estomatológica que pode ser considerado uma doença populacional, ficando atrás apenas da cárie dentária e das doenças periodontais (Kmeid et al., 2020). Diversos estudos mostram que aproximadamente $60-70 \%$ da população possuem pelo menos algum sinal ou sintoma de DTM em algum momento da vida, porém apenas 5\% precisam de tratamento para DTM (Alahmary, 2019).

A doença causada pelo novo coronavírus (SARS-CoV-2) originou-se de um mercado em Wuhan, China, no início de dezembro de 2019. De maneira bastante rápida, a COVID-19 se espalhou em todo o mundo causando uma síndrome respiratória aguda grave de coronavírus 2 (SARS-CoV-2). (Medeiros et al., 2020).

Vários países em todo o mundo adotaram medidas restritivas e de isolamento social como forma de controlar a disseminação do vírus e não levar o sistema de saúde ao colapso (Medeiros et al., 2020). Em decorrência disso, a pandemia conduziu a uma maior predisposição ao desenvolvimento de ansiedade, depressão, distúrbios do sono e suicídio (Sher, 2020; 
Huan \& Zhao, 2020). Em um estudo realizado por Wang et al. (2020) com o objetivo de avaliar o impacto psicológico, ansiedade, depressão e estresse nos estágios iniciais da pandemia de COVID-19 concluiu que de impacto psicológico, ansiedade, mais da metade dos entrevistados classificou o impacto psicológico como moderado a grave e cerca de um terço relatou ansiedade moderada a grave.

De acordo com Saccomano et al. (2020) diversos estudos relataram a associação entre aspectos psicológicos e os sintomas de DTM tanto no início quanto na progressão. A ansiedade e o estresse podem atuar aumentando tanto a frequência, quando a intensidade e a duração dos hábitos parafuncionais, sendo responsáveis pela hiperatividade dos músculos mastigatórios e sobrecarga da ATM, ocasionando o surgimento dos sintomas de DTM.

A depressão e ansiedade podem conduzir ao surgimento de sintomas de DTM e enxaqueca por meio da interação com redes moduladoras da dor e diminuindo o limiar de dor ou até mesmo modificando a percepção da dor em indivíduos que sofrem de ansiedade ou depressão, embora o mecanismo exato ainda não esteja claro (Nazeri et al., 2018).

Estudos citados por Emod-Perlman i et al. (2020) e conduzidos em dois países distintos, Israel e Polônia, com objetivo de avaliar o efeito da pandemia de COVID-19 sobre a possível prevalência e piora dos sinais e sintomas de DTM e bruxismo. Assim, evidenciou-se que a pandemia contribuiu para alterações significativos no estado psicoemocional das populações israelense e polonesa, resultando na intensificação de ambas as afecções.

Outro estudo conduzido por Medeiros et al. (2020) que também avaliou a influência da pandemia de COVID-19 e suas consequências nos sintomas de DTM concluiu que o isolamento social devido à pandemia de COVID-19 tem aumentado a prevalência de sintomas de DTM, ansiedade e depressão.

\section{Considerações Finais}

A pandemia da COVID-19 pode estar associada ao aumento de sintomas psicológicos como ansiedade e depressão em virtude do período de isolamento social. Esses sintomas possuem associação as disfunções temporomandibulares e bruxismo levando ao surgimento e desenvolvimento de ambas afecções. Outros estudos epidemiológicos devem ser conduzidos para enfatizar ainda mais esta associação e possibilitar diagnósticos e tratamentos mais satisfatórios aos pacientes.

\section{Referências}

Almeida-Leite, C. M., Stuginski-Barbosa, J., \& Conti, P. (2020). How psychosocial and economic impacts of COVID-19 pandemic can interfere on bruxism and temporomandibular disorders?. Journal of applied oral science: revista FOB, 28, e20200263.

Emodi-Perlman, A., Eli, I., Smardz, J., Uziel, N., Wieckiewicz, G., Gilon E. et al. (2020). Temporomandibular Disorders and Bruxism Outbreak as a Possible Factor of Orofacial Pain Worsening during the COVID-19 Pandemic-Concomitant Research in Two Countries. Journal Of Clinical Medicine. 9(10), 3250, 2020 .

Huang, Y., \& Zhao, N. (2020). Generalized anxiety disorder, depressive symptoms and sleep quality during COVID-19 outbreak in China: a web-based crosssectional survey. Psychiatry research, 288, 112954.

Kmeid, E., Nacouzi, M., Hallit, S., \& Rohayem, Z. (2020). Prevalence of temporomandibular joint disorder in the Lebanese population, and its association with depression, anxiety, and stress. Head \& face medicine, 16(1), 19.

Li Q., Guan, X., Wu, P., Wang, X., Zhou, L., Tong, Y. et al. (2020). Early Transmission Dynamics in Wuhan, China, of Novel Coronavirus-Infected Pneumonia. N Engl J Med. 382(13), 1199-1207.

Machado, N., Costa, YM, Quevedo, HM, Stuginski-Barbosa, J., Valle, CM, Bonjardim, LR, Garib, DG, \& Conti, P. (2020). The association of self-reported awake bruxism with anxiety, depression, pain threshold at pressure, pain vigilance, and quality of life in patients undergoing orthodontic treatment. Revista de ciências orais aplicadas: revista FOB , 28 , e20190407.

Medeiros, R. A., Vieira, D. L., Silva, E., Rezende, L., Santos, R., \& Tabata, L. F. (2020). Prevalence of symptoms of temporomandibular disorders, oral behaviors, anxiety, and depression in Dentistry students during the period of social isolation due to COVID-19. Journal of applied oral science: revista FOB, 28 , e20200445.

Nazeri, M., Ghahrechahi, H.R., Pourzare, A., Abareghi, F., Samiee-Rad, S., Shabani, M., Arjman, S., Abazarpour, R. (2018). Role of anxiety and depression in association with migraine and myofascial pain temporomandibular disorder. Indian J Dent Res, 29(5), 583-587. 
Research, Society and Development, v. 10, n. 2, e6110212609, 2021

(CC BY 4.0) | ISSN 2525-3409 | DOI: http://dx.doi.org/10.33448/rsd-v10i2.12609

O Estadão. (2020). Bruxismo e fraturas: dentistas contam as sequelas da quarentena na boca dos brasileiros.

Organização Mundial da Saúde - OMS. (2020). OMS anuncia surto de COVID-19 como uma pandemia [internet]. Copenhagen: WHO.

Przystańska, A., Jasielska, A., Ziarko, M., Pobudek-Radzikowska, M., Maciejewska-Szaniec, Z., Prylińska-Czyżewska, A., Wierzbik-Strońska, M., Gorajska, M., \& Czajka -Jakubowska, A. (2019). Psychosocial Predictors of Bruxism. BioMed research international, 2069716.

Quadri, M. F., Mahnashi, A., Al Almutahhir, A., Tubayqi, H., Hakami, A., Arishi, M., \& Alamir, A. (2015). Association of Awake Bruxism with Khat, Coffee, Tobacco, and Stress among Jazan University Students. International journal of dentistry, $2015,842096$.

Saccomanno, S., Bernabei, M., Scoppa, F., Pirino, A., Mastrapasqua, R., \& Visco, MA (2020). Coronavirus Lockdown as a Major Life Stressor: Does It Affect TMD Symptoms? International Journal of Environmental Research and Public Health. 17(23), 8907.

Sher L. (2020). COVID-19, anxiety, sleep disturbances and suicide. Sleep medicine, 70, 124.

Wang, C., Pan, R., Wan, X., Tan, Y., Xu, L., Ho, C. S., \& Ho, R. C. (2020). Immediate Psychological Responses and Associated Factors during the Initial Stage of the 2019 Coronavirus Disease (COVID-19) Epidemic among the General Population in China. International journal of environmental research and public health, 17(5), 1729. 